TITLE:

\title{
A pre-targeting strategy for MR imaging of functional molecules using dendritic Gd-based contrast agents.
}

\section{AUTHOR(S):}

Sano, Kohei; Temma, Takashi; Azuma, Takashi; Nakai, Ryusuke; Narazaki, Michiko; Kuge, Yuji; Saji, Hideo

\section{CITATION:}

Sano, Kohei ... [et al]. A pre-targeting strategy for MR imaging of functional molecules using dendritic Gd-based contrast agents.. Molecular imaging and biology 2011, 13(6): 1196-1203

\section{ISSUE DATE:}

2011-12

URL:

http://hdl.handle.net/2433/153055

\section{RIGHT:}

The final publication is available at www.springerlink.com; この論文は 出版社版でありません。引用の際には出版社版をご確認ご利用くださ $\omega_{\circ}$; This is not the published version. Please cite only the published version. 


\section{Title Page:}

A Pre-targeting Strategy for MR Imaging of Functional Molecules Using Dendritic Gd-Based Contrast Agents

Kohei Sano ${ }^{1}$, Takashi Temma ${ }^{1}$, Takashi Azuma ${ }^{2}$, Ryusuke Nakai ${ }^{2}$, Michiko Narazaki ${ }^{3}$, Yuji Kuge $^{1,4}$, Hideo Saji ${ }^{1}$

${ }^{1}$ Department of Patho-Functional Bioanalysis, Graduate School of Pharmaceutical Sciences, Kyoto University, Kyoto 606-8501, Japan

${ }^{2}$ Department of Medical Simulation Engineering Research Center for Nano Medical Engineering Institute for Frontier Medical Sciences, Kyoto University, Kyoto 606-8501, Japan

${ }^{3}$ Department of Systems Science, Graduate School of Informatics, Kyoto University, Kyoto 606-8501, Japan

${ }^{4}$ Central Institute of Isotope Science, Hokkaido University, Sapporo 060-8638, Japan

\section{Corresponding author:}

Hideo Saji, PhD

Department of Patho-Functional Bioanalysis 
Graduate School of Pharmaceutical Sciences, Kyoto University

46-29 Yoshida Shimoadachi-cho, Sakyo-ku, Kyoto 606-8501, Japan

Tel; (+81/0)-75-753-4556

Fax; (+81/0)-75-753-4568

E-mail; hsaji@pharm.kyoto-u.ac.jp

\section{Running Title:}

MRI protocol for molecular imaging by a pre-targeting method

\section{Manuscript category}

Article 


\section{ABSTRACT ( 150 words)}

Purpose

We aimed to establish a magnetic resonance imaging (MRI) protocol for the sensitive and specific imaging of functional molecules with a pre-targeting strategy utilizing the streptavidin-biotin interaction. Membrane type-1 matrix metalloproteinase (MT1-MMP) was selected as the target molecule.

\section{Procedures}

The biotinylated polyamidoamine dendrimer (PAMAM)-based contrast agent (Bt-PAMAM-DTPA(Gd)) was prepared, and its proton relaxivity ( $r 1)$ and affinity to streptavidin were evaluated. Tumor-bearing mice were pre-targeted with streptavidin-conjugated anti-MT1-MMP monoclonal antibody (mAb), streptavidin-conjugated negative control IgG, or saline and 3 days later were injected with Bt-PAMAM-DTPA(Gd) followed immediately by MRI for a period of $3 \mathrm{~h}$.

Results

High $r 1$ (15.5 $\left.\mathrm{L} \mathrm{mmol}^{-1} \mathrm{~s}^{-1}\right)$ and 1.9-fold higher affinity than D-biotin were obtained. Significantly higher relative tumor signals were observed in mice pre-targeted with streptavidin-conjugated anti-MT1-MMP mAb (165\% at 3 h vs. pre-administration) than with saline or streptavidin-conjugated negative control $\operatorname{IgG}(P<0.0001)$.

Conclusions 
This pre-targeting approach can accomplish sensitive and specific in vivo MRI of functional molecules.

\section{Key Words}

Pre-targeting, Polyamidoamine dendrimer (PAMAM), Membrane type-1 matrix metalloproteinase, Magnetic Resonance Imaging 


\section{INTRODUCTION}

Magnetic resonance imaging (MRI), characterized by a remarkable spatial resolution, is a powerful tool for noninvasive morphologic diagnosis of diseases including cancer. Recently, the application of MRI to functional molecular imaging coupled with anatomical information has been explored. To realize functional molecular imaging by MRI, contrast agents are required that possess a high relaxation to produce high MR signals to compensate for the low intrinsic sensitivity of MRI [1] in addition to selectively accumulating in the targeting site.

Gadolinium (Gd) chelates conjugated to macromolecules such as liposomes, micelles and dendrimers can give rise to enhanced proton relaxivities in comparison with simple, small molecule contrast agents such as Gd-diethylenetriamine pentaacetic acid (Gd-DTPA) [2-4]. This effect is due to a restriction in thermal flexibility leading to increased interactions between the Gd atom and surrounding water molecules [5-7]. Some groups have developed monoclonal antibody (mAb)-conjugated macromolecular contrast agents for imaging integrin $\alpha v \beta 3$ or human epidermal growth factor receptor type 2 (HER2) in tumors, which successfully increased the tumor signal intensity by 15-30\% [8, 9]. However, many researchers have failed to demonstrate in vivo functional molecular imaging using macromolecules conjugated with several Gd chelates and targeting moieties like antibodies and peptides because the pharmacokinetics of the targeting moieties were significantly altered 
by the introduction of macromolecular contrast agents, which resulted in low target recognition and high accumulation of the labeling agent in non-targeted tissues $[10,11]$. Furthermore, when antibody-conjugated macromolecular contrast agents are injected at a Gd dose $(0.1 \mathrm{mmol} \mathrm{Gd} / \mathrm{kg}$ ) necessary for adequate imaging, excess antibody (on the order of milligrams per mouse) are typically administered, which leads to major limitations of cost and in vivo toxicity.

Thus, in this study, to overcome these problems and to realize functional molecular MRI by a macromolecule-based contrast agent, we aimed to use a pre-targeting strategy that utilizes the high affinity interaction between streptavidin and biotin $\left(K d=10^{-15} \mathrm{M}\right)$ [12]. In this pre-targeting method, the first step is to administer a streptavidin-conjugated target-specific antibody. In the second step, after selective accumulation of streptavidin-conjugated antibody in the targeted tissue and clearance of unbound targeting agent from the circulation, a biotin-bound imaging probe is injected. As the post-administration contrast agent, polyamideamine dendrimer (PAMAM) was selected as the base structure since it is structurally well-defined and functional moieties including biotins and Gd chelates for both targeting and signal emission functions can easily be attached to the large number of its surface amino groups. The pre-targeting strategy is expected to provide selective and effective accumulation of the PAMAM-based contrast agent to the targeted site and a high $\mathrm{S} / \mathrm{N}$ ratio during the first hours following administration as has been observed in 
radioimmunotherapy and radioimmunodetection [13-16], and to potentially lead to lower in vivo toxicity $[17,18]$.

Thus, in this paper we describe our efforts to establish a sensitive and specific in vivo MRI protocol for imaging functional molecules utilizing a pre-targeting strategy that combines a streptavidin-conjugated antibody with a PAMAM based contrast agent modified with biotins. As the targeted biomolecule, membrane type- 1 matrix metalloproteinase (MT1-MMP) was selected. Since MT1-MMP is exclusively expressed in tumors and is closely associated with metastasis [19] and invasion [20], MT1-MMP is a potential imaging target for evaluation of tumor malignancy. 


\section{MATERIALS AND METHODS}

Synthesis of streptavidin-conjugated anti-MT1-MMP mAb

Streptavidin-conjugated anti-MT1-MMP mAb and streptavidin-conjugated negative control IgG were synthesized according to a previously described method [14]. Briefly, EZ-Link $^{\circledR}$ sulfosuccinimidyl-6-(biotinamido) hexanoate (sulfo-NHS-LC-biotin) (Pierce, Inc.) was added to a solution of anti-MT1-MMP mAb (113-5B7, Daiichi Fine Chemical Co.) in a molar ratio of 12:1. The mixture was gently stirred for $30 \mathrm{~min}$ at room temperature and then was purified with a diafiltration membrane (Amicon Ultra 4 (MWCO 30,000), Millipore Co.). A solution of biotinylated anti-MT1-MMP mAb was added to a solution of streptavidin (SAv; Pierce, Inc.) in a 1:3 molar ratio. The mixture was incubated for $1 \mathrm{~h}$ at $37^{\circ} \mathrm{C}$ followed by purification twice by affinity chromatography using a HiTrap rProtein A column (GE Healthcare Bioscience). The eluate containing anti-MT1-MMP mAb-SAv was concentrated with a diafiltration membrane (MWCO 30,000), and the protein concentration was determined by the bicinchoninate (BCA) method. The purification was monitored by a size exclusion chromatograph using a 300×4.6-mm i.d. TSK-Gel Super SW 3000 column (Tosoh Co., Japan) eluted with phosphate buffer $(0.1 \mathrm{M}, \mathrm{pH}$ 6.8) at a flow rate of $0.1 \mathrm{~mL} / \mathrm{min}$. Comparison of molecular mass standards (Oriental Yeast Co., Japan) of the absorbance at $280 \mathrm{~nm}$ indicated that a peak at 30.3 min was consistent with the presence of the $210 \mathrm{kDa}$ streptavidin-conjugated anti-MT1-MMP mAb. Furthermore, streptavidin-conjugated 
anti-MT1-MMP mAb retained 81.3\% immunoreactivity of the anti-MT1-MMP mAb, which was confirmed by flow cytometry.

Synthesis of Bt-PAMAM-DTPA(Gd)

EZ-Link $^{\circledR}$ sulfo-NHS-LC-biotin was added to a solution of PAMAM (generation 4 (G4))

(Sigma Aldrich) in a molar ratio of 20:1. The mixture was stirred for $30 \mathrm{~min}$ at room temperature and then was applied to a diafiltration membrane (MWCO 10,000) to remove unbound biotins as well as to change the buffer to phosphate buffer (0.1 M, pH 9.0). After purification, the incorporation ratio of biotins conjugated to each dendrimer was measured using an $\mathrm{EZ}^{\mathrm{TM}}$ Biotin Quantitation Kit (Pierce, Inc.). The biotinylated PAMAM was reacted with a 64-fold molar excess of 2-(p-isothiocyanatobenzyl)-diethylenetriaminepentaacetic acid (p-SCN-Bz-DTPA) (Macrocyclics) at $40^{\circ} \mathrm{C}$ for $24 \mathrm{~h}$. During the reaction, the $\mathrm{pH}$ was maintained at 9.0 with $1 \mathrm{~N} \mathrm{NaOH}$. An additional equal amount of p-SCN-Bz-DTPA was added after $24 \mathrm{~h}$ and the reaction was incubated for another $24 \mathrm{~h}$ at $40^{\circ} \mathrm{C}$. The resulting preparation was purified by diafiltration membrane (MWCO 10,000). After purification, the number of DTPAs incorporated into each G4 dendrimer was checked by chelate titration using $\mathrm{ZnSO}_{4}$ (indicator: 4-(2-pyridylazo)resorcinol, $\mathrm{NH}_{3} / \mathrm{NH}_{4}{ }^{+}, \mathrm{pH}$ 10) according to a previously described method with some modification [21]. Purified Bt-PAMAM-DTPA was mixed with $\mathrm{GdCl}_{3}$ (Sigma Aldrich) in citrate buffer $(0.3 \mathrm{M}, \mathrm{pH} 5.0)$ for $2 \mathrm{~h}$ at $40^{\circ} \mathrm{C}$. The 
excess Gd was removed by diafiltration membrane (MWCO 10,000) while simultaneously changing the buffer to PBS (0.1 M, pH 7.4). The number of Gd incorporated into a dendrimer was checked by separating the free Gd and Bt-PAMAM-DTPA(Gd) with diafiltration filter after labeling the Bt-PAMAM-DTPA with ${ }^{153} \mathrm{Gd}$ and nonradioactive Gd. For comparison purposes, PAMAM-DTPA(Gd) containing one biotin (Bt $t_{1}$-PAMAM-DTPA(Gd)) was also prepared in a similar manner.

Stability of Bt-PAMAM-DTPA(Gd) in mouse plasma

${ }^{153}$ Gd-labeled Bt-PAMAM-DTPA(Gd) was prepared by reacting Bt-PAMAM-DTPA(Gd) with ${ }^{153} \mathrm{Gd}(1 \mu \mathrm{Ci}$, PerkinElmer Japan Co., Osaka, Japan) and non-radioactive $\mathrm{Gd}$ in $0.3 \mathrm{M}$ citrate buffer at $\mathrm{pH} 5.0$ for $2 \mathrm{~h}$ at $40^{\circ} \mathrm{C}$. ${ }^{153} \mathrm{Gd}$ labeled Bt-PAMAM-DTPA(Gd) (30 $\mu \mathrm{L})$ was added to mouse plasma collected from female $\mathrm{C} 3 \mathrm{H} / \mathrm{He}$ mice $(270 \mu \mathrm{L})$, and the plasma samples were incubated at $37^{\circ} \mathrm{C}$ for 0,3 , and $24 \mathrm{~h}$. After incubation, aliquots of the samples were drawn, and radioactivity was analyzed by size-exclusion chromatography with a PD-10 column (GE Healthcare Bioscience) using saline as eluent.

Affinity of Bt-PAMAM-DTPA(Gd) for streptavidin

Competition assays of ${ }^{125} \mathrm{I}$-(3-iodobenzoyl)norbiotinamide $\left({ }^{125} \mathrm{I}-\mathrm{IBB}\right)$, a radiolabeled 
biotin derivative synthesized as reported previously [22], were performed by incubating streptavidin (Pierce, Inc.; $400 \mu \mathrm{L}, 2 \mu \mathrm{g} / \mathrm{mL}$ ), ${ }^{125} \mathrm{I}-\mathrm{IBB}(50 \mu \mathrm{L}, 5 \mu \mathrm{Ci}$ ), and various concentrations of Bt-PAMAM-DTPA(Gd), Bt ${ }_{1}$-PAMAM-DTPA(Gd), and D-biotin (Nacalai Tesque, Kyoto, Japan) (50 $\left.\mu \mathrm{L}, 10^{-8} \sim 10^{-4} \mathrm{M}\right)$ in PBS (0.1 M, pH 7.4) for $60 \mathrm{~min}$ at $37^{\circ} \mathrm{C}$. At the end of the incubation, the mixture was applied to a size exclusion column with a Sephadex G-50 Fine (GE Healthcare Bioscience), followed by measurement of the radioactivity from the column eluent (containing macromolecules) with a NaI well-type scintillation counter (1470WIZARD, PerkinElmer Japan Co.). Nonspecific binding was determined in the presence of $10 \mathrm{mg} / \mathrm{mL}$ D-biotin. The $50 \%$ inhibitory concentrations $\left(\mathrm{IC}_{50} \mathrm{~S}\right)$ were determined from displacement curves of the percent inhibition of ${ }^{125}$ I-IBB binding $v s$. the inhibitor concentration.

Preparation of tumor-bearing animals

Female C3H/He mice (5 weeks old), supplied by Japan SLC Co. (Hamamatsu, Japan), were housed under a 12-h light/12-h dark cycle and were given free access to food and water. The animal experiments in this study were conducted in accordance with institutional guidelines and were approved by the Kyoto University Animal Care Committee, Japan.

FM3A mouse breast carcinoma cells were supplied by the Health Science Research Resources Bank (Osaka, Japan). They were cultured in DMEM medium (Nissui 
Pharmaceutical Co.) supplemented with $10 \%$ fetal bovine serum at $37^{\circ} \mathrm{C}$ in a humidified atmosphere containing 5\% $\mathrm{CO}_{2}$ and $95 \%$ air and had a 10.6-h doubling time.

FM3A cells were suspended in $0.01 \mathrm{M}$ PBS ( $\mathrm{pH}$ 7.4) followed by subcutaneous inoculation into the right hind leg of the mouse $\left(5 \times 10^{6}\right.$ cells $\left./ 100 \mu \mathrm{L} / \mathrm{mouse}\right)$ [23]. The tumor volume was estimated by (length) $\times(\text { width })^{2} / 2$ [24] over a 10-day tumor growth period. The average size of the tumors was $213 \pm 82 \mathrm{~mm}^{3}$ on the MR imaging study day. The expression of MT1-MMP in FM3A cells and tumor tissues was confirmed by western blotting and immunohistochemistry [25].

\section{Magnetic Resonance Imaging}

MRI was performed using a clinical 1.5 Tesla MR scanner (MAGNETOM Symphony Sonata, Siemens). All $T_{1}$-weighted $\mathrm{MR}$ images were acquired with a multislice spin-echo pulse sequence. MRI data were analyzed using the ImageJ software.

\section{Phantom study}

Solutions of Gd-DTPA (Sigma Aldrich) and Bt-PAMAM-DTPA(Gd) were prepared with a Gd concentration in the range of 10 to $500 \mu \mathrm{M}$ in vials with an inner diameter of 15 $\mathrm{mm}$ followed by the MR scan using a knee coil $\left(20.5 \mathrm{~cm}\right.$ in diameter) at $20^{\circ} \mathrm{C}$. To obtain proton relaxivity $(r 1)$ for samples, spin-echo images were obtained using a sequence with $\mathrm{TR}=500,1000,1500$, and $2000 \mathrm{msec}$ and with $\mathrm{TE}=15 \mathrm{msec}$. The imaging parameters were as 
follows: field-of-view, $256 \times 128 \mathrm{~mm}$; matrix, $256 \times 128$; slice thickness, $7 \mathrm{~mm}$; number of average, 3 .

In vivo study

Mice ( $n=4)$ bearing FM3A tumors in the right thigh received streptavidin-conjugated anti-MT1-MMP mAb (50 $\mu \mathrm{g} / 100 \mu \mathrm{L}$ in saline) via tail vein. Three days later, Bt-PAMAM-DTPA(Gd) (0.1 mmolGd/kg, $100 \mu \mathrm{L}$ in PBS (0.1 M, pH 7.4), i.v.) was injected followed by data acquisition by MRI at several time points over a 3-h post-injection period under sodium pentobarbital (50 mg/kg, i.p.) anesthesia. All MR images were obtained using a hand-made round surface coil (5.5 cm in diameter) fixed by a custom constructed coil holder. The imaging parameters were as follows: TR/TE, 300/5.2 msec; field-of-view, 128×96 mm; matrix, 256×192; slice thickness, $1.5 \mathrm{~mm}$; number of average, 3. MRI studies were also conducted as above on FM3A tumor bearing mice $(n=3)$ pre-treated with saline $(100 \mu \mathrm{L})$ or streptavidin-conjugated negative control IgG (50 $\mu \mathrm{g} / 100 \mu \mathrm{L}$ in saline). The signal intensity was calculated by drawing a region of interest around the tumor, muscle in the contralateral hind limb, and kidneys. The relative signal intensity in each tissue was defined as the signal intensity after administration of Bt-PAMAM-DTPA(Gd) divided by the signal intensity before administration. 
Unpaired Student's $t$ test was used to evaluate the significance of differences of $r 1$ between Bt-PAMAM-DTPA(Gd) and Gd-DTPA. To compare the time courses of relative signal intensity in the tumor and kidneys and tumor/muscle (T/M) signal ratios among Bt-PAMAM-DTPA(Gd) pre-targeted by streptavidin-conjugated anti-MT1-MMP mAb, streptavidin-conjugated negative control IgG, and saline, two-way repeated measures ANOVA with post-hoc analysis by the Tukey-Kramer test was performed. Differences at the 95\% confidence level $(P<0.05)$ were considered significant. 


\section{RESULTS}

Characterization of Bt-PAMAM-DTPA(Gd)

Bt-PAMAM-DTPA(Gd) was synthesized in four steps from PAMAM in a yield of

63\%. PAMAM was conjugated to $9.9 \pm 1.3$ biotins and $43.6 \pm 1.9$ DTPAs, which were quantitatively coordinated to $\mathrm{Gd}$. $\mathrm{Bt}_{1}$-PAMAM-DTPA(Gd) containing $1.0 \pm 0.1$ biotin and $47.6 \pm 2.2$ DTPAs on PAMAM dendrimer was also synthesized. The ${ }^{153}$ Gd-labeled Bt-PAMAM-DTPA(Gd), which was incubated with mouse plasma for $24 \mathrm{~h}$, did not release any low molecular weight metabolites or free radiometals (Fig. 1).

The competitive binding assay revealed that all of the contrast agents inhibited the binding of ${ }^{125} \mathrm{I}-\mathrm{IBB}$ to streptavidin in a dose-dependent manner (Fig. 2). The $\mathrm{IC}_{50} \mathrm{~S}$ for Bt-PAMAM-DTPA(Gd), Bt 1 -PAMAM-DTPA(Gd), and D-biotin were $32 \pm 31,1390 \pm 1220$, and $60 \pm 45 \mathrm{nM}$, respectively, demonstrating that Bt-PAMAM-DTPA(Gd) had about 1.9- and 43.2-fold higher affinity to streptavidin than D-biotin and Bt $\mathrm{B}_{1}$-PAMAM-DTPA(Gd).

MR imaging study (Phantom study)

The in vitro $T_{1}$-weighted MR images with Bt-PAMAM-DTPA(Gd) and Gd-DTPA are shown in Fig. 3a. Water and PBS were used as baselines. With the same Gd concentration, the signals with Bt-PAMAM-DTPA(Gd) were higher compared to Gd-DTPA. The longitudinal relaxation rate $\left(1 / T_{1}\right)$ vs. the concentration of $\mathrm{Gd}$ for both contrast agents are 
shown in Fig. 3b with good linear fits $\left(\mathrm{R}^{2}=1.00\right.$ and 0.99 for Bt-PAMAM-DTPA(Gd) and Gd-DTPA, respectively). Calculated $r 1$ values $\left(\mathrm{L} \mathrm{mmol}^{-1} \mathrm{~s}^{-1}\right)$ for Bt-PAMAM-DTPA(Gd) and Gd-DTPA were $15.5 \pm 1.1$ and $3.6 \pm 0.1$, respectively, which shows that the proton relaxivity of Bt-PAMAM-DTPA(Gd) was 4.3-fold higher than that of Gd-DTPA $(P<0.0001)$.

MR imaging study (in vivo study)

Figure 4a (coronal) and 4b (transaxial) show in vivo $T_{1}$-weighted $\mathrm{MR}$ images of tumor-bearing mice before and at $5 \mathrm{~min}$ and $180 \mathrm{~min}$ after injection of Bt-PAMAM-DTPA(Gd) following pre-treatment with streptavidin-conjugated anti-MT1-MMP mAb (MT1-MMP), streptavidin-conjugated negative control IgG (Negative Control), or saline (Saline). In the MT1-MMP group, the most intense signal was observed in the margin of the tumor, as compared with the tumor core, over the 180-min period. The relative signal intensity (rSI) in the tumor and the relative T/M ratio were strongly enhanced just after administration of Bt-PAMAM-DTPA(Gd) and were highly maintained for $3 \mathrm{~h}$ after the contrast agent injection as well as the rapid clearance from the circulation (Fig. 4c, d). These signals were significantly greater than those from the Negative Control group $(P<$ 0.05 (at 44 and $55 \mathrm{~min}$ ), $P<0.01$ (at 2 and $3 \mathrm{~h}$ )). In the Saline group, Bt-PAMAM-DTPA(Gd) readily disappeared from the circulation and mainly accumulated in the kidneys. Though the relative signal intensity in the tumor also increased (190\%) just after administration of 
Bt-PAMAM-DTPA(Gd), it decreased to the basal level (115\%) within 3 h (Fig. 4c). A slightly higher tumor signal was obtained in the Negative Control group than in the Saline group only $3 \mathrm{~h}$ after injection of Bt-PAMAM-DTPA(Gd) $(\mathrm{P}<0.01)$. The time-dependent change of relative T/M ratios was similar to that of the relative signal intensity in the tumor (Fig. 4d). The time-dependent change of relative signal intensity in the kidneys was very similar in all three groups (Fig. 4e). 


\section{DISCUSSION}

In this study, we accomplished visualization of MT1-MMP by MRI using a pre-targeting method with a PAMAM-based contrast agent (Bt-PAMAM-DTPA(Gd)) which possesses high proton relaxivity and high affinity to streptavidin. For future applications, this pre-targeting method based on the interaction between biotin and streptavidin is promising for the detection of functional molecules, such as biomarkers in tumors like MT1-MMP, by in vivo MRI.

Although several macromolecular contrast agents have been developed for functional molecular imaging with MRI using a mAb or peptide as the targeting moiety, these attempts have been largely unsuccessful because the macromolecular contrast agents, such as an antibody attached to a dendrimer, have a poorer targeting ability and slower pharmacokinetics in the circulation than the targeting moiety alone, which leads to an inadequately low S/N ratio for several days post-injection $[10,11]$. Thus, we focused on a pre-targeting strategy whose effectiveness in elevating the $\mathrm{S} / \mathrm{N}$ ratio shortly after injection has been well documented in the field of radioimmunotherapy $[13,26]$. In the pre-targeting strategy, high affinity between the pre- and post-administered agents is required; thus, the affinity of a post-administered biotinylated contrast agent to streptavidin needed to be evaluated. In this study, Bt-PAMAM-DTPA(Gd) containing approximately 10 biotins in the structure showed 43.2- and 1.9-fold higher affinity to streptavidin compared with $\mathrm{Bt}_{1}$-PAMAM-DTPA(Gd) 
containing only one biotin per dendrimer and D-biotin, respectively, which suggests a multivalent effect of Bt-PAMAM-DTPA(Gd) binding to streptavidin. Zhu et al. recently reported the MRI of functional molecules by a pre-targeting approach [27]; however, the authors failed to show a significant tumor image probably because of the small number of biotins per dendrimer ( $\sim 4$ biotins per dendrimer). Therefore, in a pre-targeting method where a macromolecule is used as the post-administered agent, it is essential that an optimal number of biotins on the macromolecule is evaluated.

In the streptavidin-conjugated anti-MT1-MMP mAb-treated group, MR signals in the tumor and T/M ratios were highly maintained following Bt-PAMAM-DTPA(Gd) administration compared with the saline-treated group, which suggests that the tumor accumulation of Bt-PAMAM-DTPA(Gd) depended on the pre-targeted streptavidin-conjugated anti-MT1-MMP mAb. Furthermore, MR signals in the tumor and $\mathrm{T} / \mathrm{M}$ ratios were also significantly higher in the streptavidin-conjugated anti-MT1-MMP mAb-treated group than those in the negative control, which suggests that the accumulation of Bt-PAMAM-DTPA(Gd) was primarily specific for MT1-MMP. The slightly significant difference in the relative tumor signal was shown between negative control and saline group is probably caused in part by passive accumulation of the pre-targeted streptavidin-conjugated antibody as a macromolecule due to an enhanced permeability and retention effect [28]. 
Previously, to determine the optimal interval between injections of streptavidin-conjugated anti-MT1-MMP mAb and Bt-PAMAM-DTPA(Gd), the biodistribution of ${ }^{125}$ I-labeled streptavidin-conjugated anti-MT1-MMP mAb was evaluated in C3H/He mice bearing FM3A mouse breast carcinoma [14]. From consideration of the high accumulation of streptavidin-conjugated antibody in the tumor and the high tumor to blood ratio at $72 \mathrm{~h}$, we adopted this time as the interval between pre- and post-administrations in this study. Recently, some reports have shown that clearing agents (e.g. galactosylated biotin-albumin conjugate) can readily (within a few hours) clear surplus streptavidin conjugated antibody in the circulation to the liver where the complex is metabolized and excreted without loss of the biotin binding sites in the tumor [29-31], thereby shortening the interval between injections. In the future, by taking advantage of this type of strategy, we can establish an optimal protocol for MT1-MMP imaging for clinical applications.

Dendrimers are a class of highly branched spherical polymers, with a variety of properties, such as chemical structure, size, molecular weight and functional groups that can be easily manipulated at the molecular level through their synthesis. The pharmacokinetics of the dendrimer is susceptible to control with its generation number such that it may be highly bioavailable, an important consideration for a variety of applications, especially in the biomedical field [32]. Here, PAMAM dendrimer was chosen as the base structure of the contrast agent for post-administration. PAMAM dendrimer (G4) with an ethylene diamine 
core has a molecular weight of 14,215 Da and possesses 64 amino groups on the surface of the molecule [33]. In this study, 10 biotins and 44 Gds for specific targeting and sensitive imaging were introduced onto the dendrimer. An in vitro MR study showed that the relaxivity of Bt-PAMAM-DTPA(Gd) was 4.3-fold higher than Gd-DTPA, which indicates the effectiveness of Bt-PAMAM-DTPA(Gd) as a contrast agent with high proton relaxivity as expected because of slow tumbling rates and a short water residence time $[2,5]$.

The post-administered contrast agent in a pre-targeting study should satisfy the following two requirements besides specific affinity to pre-administered streptavidin: rapid blood clearance and low nonspecific accumulation in the tumor. It has been reported that a PAMAM (G4) dendrimer is quickly excreted via glomerular filtration primarily during the first pass (the blood $\alpha$ phase half-life: $2.5 \mathrm{~min}, \beta$ phase half-life: $35 \mathrm{~min}$ [34]), and not via the bile pathway. In addition, these dendrimers exhibit no measurable leakage from normal blood vessels because of their moderate size (ca $6 \mathrm{~nm}$ ) [2, 34-37], which leads to low nonspecific accumulation in the tumor caused by passive accumulation based on an enhanced permeability and retention effect. PAMAM (G4)-based MR contrast agents can be effective as imaging probes, as supported by the experimental data that showed low MR signals observed in the tumors of the saline pre-targeted group while intense signals were observed in the kidneys after the acute disappearance of Bt-PAMAM-DTPA(Gd) from the circulation.

As mentioned above, in the case of antibody-conjugated dendrimer-based contrast 
agents, excess antibodies (on the order of milligrams per mouse) are typically administered when injected at a $\mathrm{Gd}$ dose $(0.1 \mathrm{mmol} \mathrm{Gd} / \mathrm{kg})$ necessary for adequate imaging, which leads to major limitations of cost and toxicity. On the other hand, our pre-targeting strategy could control the amount of injected streptavidin-conjugated antibody by corresponding to the targeted molecule (about $50 \mu \mathrm{g}$ per mouse for MT1-MMP), which would be useful for reducing the cost and toxicity of the imaging process.

In the application of dendrimers in vivo, cytotoxicity is often a major issue. To date, as has been widely demonstrated for other polycations, dendrimers bearing amino termini display concentration- and commonly generation-dependent cytotoxicity [38] and potent hemolytic activity [39]. These effects could be attributable to the electrostatic interactions of the positively charged dendrimer with the negatively charged cell membrane under physiological pH. Nevertheless, Bt-PAMAM-DTPA(Gd) used in this study was negatively charged due to modifications of the amino termini to bind biotin and DTPA such that it could be acceptable in vivo. This assertion is supported by a report that PAMAM dendrimers bearing carboxylate termini display dramatically lower toxicity to cells [40]. We also plan to acetylate or succinylate the free amino groups to further reduce the positive charge of the complexes if needed to decrease toxicity and hemolytic activity. The rapid excretion of Bt-PAMAM-DTPA(Gd) via glomerular filtration should alleviate adverse effects such as nephrogenic systemic fibrosis [41] derived from released Gd, as compared with 
macromolecular contrast agents which have slow elimination pharmacokinetics [11, 42], although further analysis of the cytotoxicity is needed. 


\section{CONCLUSIONS}

The pre-targeting method utilizing the specific interaction between streptavidin and biotin enabled the visualization of MT1-MMP expressing tumors by $1.5 \mathrm{~T}$ MRI with high $\mathrm{S} / \mathrm{N}$ ratios during the first hours following administration of a contrast agent, Bt-PAMAM-DTPA(Gd). The results suggest that this method may be beneficial to diagnose tumor malignancy in a clinical setting. In future work, this method could be applied to the imaging of a variety of pathologic functional molecules expressed on cell surface. 


\section{ACKNOWLEDGMENTS}

This study was supported by Grants-in-Aid for Scientific Research and by the 21st

Century Center of Excellence Programs at Kyoto University "Knowledge Information

Infrastructure for Genome Science” from the Ministry of Education, Culture, Sports, Science and Technology, Japan. A part of this study was conducted as a part of the project, "R\&D of Molecular Imaging Equipment for Malignant Tumor Therapy Support”, supported by the New Energy and Industrial Technology Development Organization (NEDO), Japan. 


\section{Conflict of interest}

The authors have no conflict of interest. 


\section{REFERENCES}

1. Caravan P (2006) Strategies for increasing the sensitivity of gadolinium based MRI contrast agents. Chem Soc Rev 35:512-523

2. Kobayashi H,Brechbiel MW (2003) Dendrimer-based macromolecular MRI contrast agents: characteristics and application. Mol Imaging 2:1-10

3. Accardo A, Tesauro D, Roscigno P et al (2004) Physicochemical properties of mixed micellar aggregates containing CCK peptides and Gd complexes designed as tumor specific contrast agents in MRI. J Am Chem Soc 126:3097-3107

4. Mulder WJ, Strijkers GJ, van Tilborg GA, Griffioen AW,Nicolay K (2006) Lipid-based nanoparticles for contrast-enhanced MRI and molecular imaging. NMR Biomed 19:142-164

5. Nicolle GM, Toth E, Schmitt-Willich H, Raduchel B,Merbach AE (2002) The impact of rigidity and water exchange on the relaxivity of a dendritic MRI contrast agent. Chemistry 8:1040-1048

6. Toth EE, Vauthey S, Pubanz D,Merbach AE (1996) Water Exchange and Rotational Dynamics of the Dimeric Gadolinium(III) Complex [BO\{Gd(DO3A)(H(2)O) $(2)]$ : A Variable-Temperature and -Pressure (17)O NMR Study(1). Inorg Chem 35:3375-3379

7. Toth E,Merbach AE (1998) Water exchange dynamics: The key for high relaxivity contrast agents in medical magnetic resonance imaging. Ach-Models in Chemistry 135:873-884

8. Sipkins DA, Cheresh DA, Kazemi MR et al (1998) Detection of tumor angiogenesis in 
vivo by alphaVbeta3-targeted magnetic resonance imaging. Nat Med 4:623-626

9. Lee JH, Huh YM, Jun YW et al (2007) Artificially engineered magnetic nanoparticles for ultra-sensitive molecular imaging. Nat Med 13:95-99

10. Boswell CA, Eck PK, Regino CA et al (2008) Synthesis, characterization, and biological evaluation of integrin alphavbeta3-targeted PAMAM dendrimers. Mol Pharm 5:527-539

11. Kobayashi H, Sato N, Saga T et al (2000) Monoclonal antibody-dendrimer conjugates enable radiolabeling of antibody with markedly high specific activity with minimal loss of immunoreactivity. Eur J Nucl Med 27:1334-1339

12. Green NM (1990) Avidin and streptavidin. Methods Enzymol 184:51-67

13. Boerman OC, van Schaijk FG, Oyen WJ,Corstens FH (2003) Pretargeted radioimmunotherapy of cancer: progress step by step. J Nucl Med 44:400-411

14. Sano K, Temma T, Kuge Y et al (2010) Radioimmunodetection of MT1-MMP relevant to tumor malignancy with pre-targeting method. Biol Pharm Bull 32:1589-1595

15. Axworthy DB, Reno JM, Hylarides MD et al (2000) Cure of human carcinoma xenografts by a single dose of pretargeted yttrium-90 with negligible toxicity. Proc Natl Acad Sci U S A 97:1802-1807

16. Paganelli G, Malcovati M,Fazio F (1991) Monoclonal antibody pretargetting techniques for tumour localization: the avidin-biotin system. International Workshop on Techniques for Amplification of Tumour Targetting. Nucl Med Commun 12:211-234 
17. Goldenberg DM, Sharkey RM, Paganelli G, Barbet J,Chatal JF (2006) Antibody pretargeting advances cancer radioimmunodetection and radioimmunotherapy. J Clin Oncol 24:823-834

18. Sharkey RM, Karacay H, Cardillo TM et al (2005) Improving the delivery of radionuclides for imaging and therapy of cancer using pretargeting methods. Clin Cancer Res 11:7109s-7121s

19. Deryugina EI,Quigley JP (2006) Matrix metalloproteinases and tumor metastasis. Cancer Metastasis Rev 25:9-34

20. Shiomi T,Okada Y (2003) MT1-MMP and MMP-7 in invasion and metastasis of human cancers. Cancer Metastasis Rev 22:145-152

21. Laus S, Sour A, Ruloff R, Toth E,Merbach AE (2005) Rotational dynamics account for pH-dependent relaxivities of PAMAM dendrimeric, Gd-based potential MRI contrast agents. Chemistry 11:3064-3076

22. Foulon CF, Alston KL,Zalutsky MR (1997) Synthesis and preliminary biological evaluation of (3-iodobenzoyl)norbiotinamide and ((5-iodo-3-pyridinyl)carbonyl)norbiotinamide: two radioiodinated biotin conjugates with improved stability. Bioconjug Chem 8:179-186

23. Kudo T, Ueda M, Kuge Y et al (2009) Imaging of HIF-1-active tumor hypoxia using a protein effectively delivered to and specifically stabilized in HIF-1-active tumor cells. J Nucl 
Med 50:942-949

24. Zhang Y, Wang C, Zhang Y,Sun M (2004) C6 glioma cells retrovirally engineered to express IL-18 and Fas exert FasL-dependent cytotoxicity against glioma formation. Biochem Biophys Res Commun 325:1240-1245

25. Temma T, Sano K, Kuge Y et al (2009) Achievement of MT1-MMP imaging shortly after radioligand administration by pretargeting strategy with SPECT. J Nucl Med 50(suppl):337P 26. Kraeber-Bodere F, Rousseau C, Bodet-Milin C et al (2006) Targeting, toxicity, and efficacy of 2-step, pretargeted radioimmunotherapy using a chimeric bispecific antibody and 131I-labeled bivalent hapten in a phase I optimization clinical trial. J Nucl Med 47:247-255 27. Zhu W, Okollie B, Bhujwalla ZM,Artemov D (2008) PAMAM dendrimer-based contrast agents for MR imaging of Her-2/neu receptors by a three-step pretargeting approach. Magn Reson Med 59:679-685

28. Iyer AK, Khaled G, Fang J,Maeda H (2006) Exploiting the enhanced permeability and retention effect for tumor targeting. Drug Discov Today 11:812-818

29. Pantelias A, Pagel JM, Hedin N et al (2007) Comparative biodistributions of pretargeted radioimmunoconjugates targeting $\mathrm{CD} 20, \mathrm{CD} 22$, and DR molecules on human B-cell lymphomas. Blood 109:4980-4987

30. Sharkey RM, Karacay H, Griffiths GL et al (1997) Development of a streptavidin-anti-carcinoembryonic antigen antibody, radiolabeled biotin pretargeting method 
for radioimmunotherapy of colorectal cancer. Studies in a human colon cancer xenograft model. Bioconjug Chem 8:595-604

31. Lin Y, Pagel JM, Axworthy D et al (2006) A genetically engineered anti-CD45 single-chain antibody-streptavidin fusion protein for pretargeted radioimmunotherapy of hematologic malignancies. Cancer Res 66:3884-3892

32. Tomalia DA, Reyna LA,Svenson S (2007) Dendrimers as multi-purpose nanodevices for oncology drug delivery and diagnostic imaging. Biochem Soc Trans 35:61-67

33. Tomalia DA, Naylor AM,Goddard WA (1990) Starburst Dendrimers - Molecular-Level Control of Size, Shape, Surface-Chemistry, Topology, and Flexibility from Atoms to Macroscopic Matter. Angewandte Chemie-International Edition in English 29:138-175

34. Kobayashi H, Sato N, Hiraga A et al (2001) 3D-micro-MR angiography of mice using macromolecular MR contrast agents with polyamidoamine dendrimer core with reference to their pharmacokinetic properties. Magn Reson Med 45:454-460

35. Sato N, Kobayashi H, Hiraga A et al (2001) Pharmacokinetics and enhancement patterns of macromolecular MR contrast agents with various sizes of polyamidoamine dendrimer cores. Magn Reson Med 46:1169-1173

36. Choyke PL, Kobayashi H (2006) Functional magnetic resonance imaging of the kidney using macromolecular contrast agents. Abdom Imaging 31:224-231

37. Kobayashi H, Brechbiel MW (2005) Nano-sized MRI contrast agents with dendrimer 
cores. Adv Drug Deliv Rev 57:2271-2286

38. Roberts JC, Bhalgat MK,Zera RT (1996) Preliminary biological evaluation of polyamidoamine (PAMAM) Starburst dendrimers. J Biomed Mater Res 30:53-65

39. Malik N, Wiwattanapatapee R, Klopsch R et al (2000) Dendrimers: relationship between structure and biocompatibility in vitro, and preliminary studies on the biodistribution of 125I-labelled polyamidoamine dendrimers in vivo. J Control Release 65:133-148

40. Jevprasesphant R, Penny J, Jalal R et al (2003) The influence of surface modification on the cytotoxicity of PAMAM dendrimers. Int J Pharm 252:263-266

41. Buhaescu I,Izzedine H (2008) Gadolinium-induced nephrotoxicity. Int J Clin Pract 62:1113-1118

42. Kobayashi H, Kawamoto S, Jo SK et al (2003) Macromolecular MRI contrast agents with small dendrimers: pharmacokinetic differences between sizes and cores. Bioconjug Chem $14: 388-394$ 


\section{Figure Captions}

\section{Figure 1}

Size exclusion analysis of ${ }^{153}$ Gd-labeled Bt-PAMAM-DTPA(Gd) radioactivity after incubation at $37^{\circ} \mathrm{C}$ in mouse plasma. The error bars represent standard deviations.

\section{Figure 2}

Inhibition of ${ }^{125}$ I-IBB binding to streptavidin by D-biotin, Bt-PAMAM-DTPA(Gd), or $\mathrm{Bt}_{1}$-PAMAM-DTPA(Gd).

\section{Figure 3}

(a) In vitro $T_{1}$-weighted $\mathrm{MR}$ measurements of different concentrations of Gd (micromolar) from Gd-DTPA and Bt-PAMAM-DTPA(Gd) in PBS at 1.5 T. PBS and water were used as references. These images show that at all concentrations, the signals are greater for Bt-PAMAM-DTPA(Gd) than for Gd-DTPA.

(b) Longitudinal relaxation rate $\left(1 / T_{1}\right)$ vs. the concentration of Gd from Gd-DTPA (crosses) and Bt-PAMAM-DTPA(Gd) (circles) in PBS at $1.5 \mathrm{~T}$ are presented with good linear fits $\left(\mathrm{R}^{2}>0.99\right)$. The $r 1$ value for Bt-PAMAM-DTPA(Gd) was 4.3 -fold higher than for Gd-DTPA. 


\section{Figure 4}

(a, b) In vivo $T_{1}$-weighted MR images of $\mathrm{C} 3 \mathrm{H} / \mathrm{He}$ mice before and at $5 \mathrm{~min}$ and $180 \mathrm{~min}$ after injection of Bt-PAMAM-DTPA(Gd) following pre-treatment with streptavidin-conjugated anti-MT1-MMP mAb (MT1-MMP), streptavidin-conjugated negative control IgG (Negative control), or saline (Saline). The coronal (a) and transaxial (b) images are shown. Arrows or dotted squares indicate the tumor site. Enlarged images of the dotted square regions are also shown.

(c-e) The dynamic change of signal intensity in the tumor by Bt-PAMAM-DTPA(Gd) (c) and relative tumor to muscle ratios (d) following pre-treatment with streptavidin-conjugated anti-MT1-MMP mAb (MT1-MMP, circles), streptavidin-conjugated negative control IgG (Negative control, squares), or saline (Saline, crosses). (e) The dynamic change of signal intensity in the kidney for each animal group. ${ }^{*} \mathrm{P}<0.05,{ }^{\S} \mathrm{P}<0.01$ vs. Negative Control; ${ }^{*} \mathrm{P}$ $<0.01$ vs. Saline; ${ }^{\dagger} \mathrm{P}<0.01$ Negative Control vs. Saline 
A Self-archived copy in

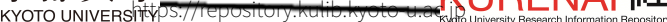

c)
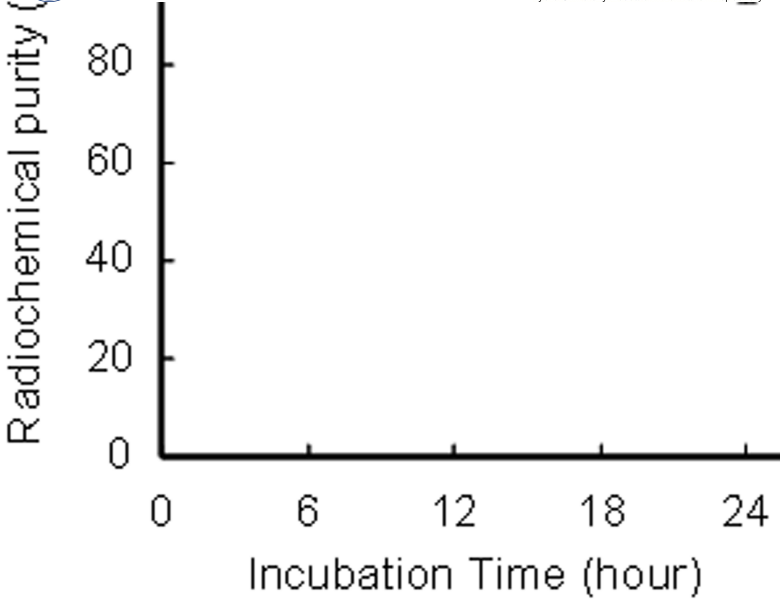


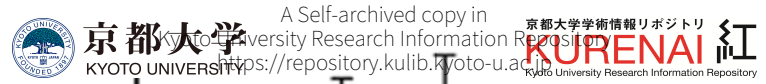

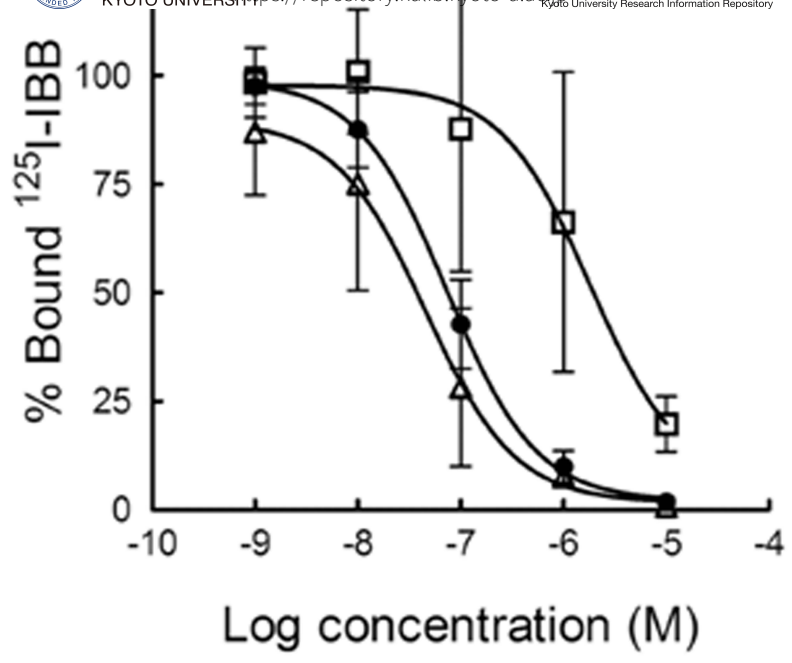

$\triangle$ Bt-PAMAM-DTPA(Gd)

- - D-biotin

$\rightarrow-B_{1}$-PAMAM-DTPA(Gd) 


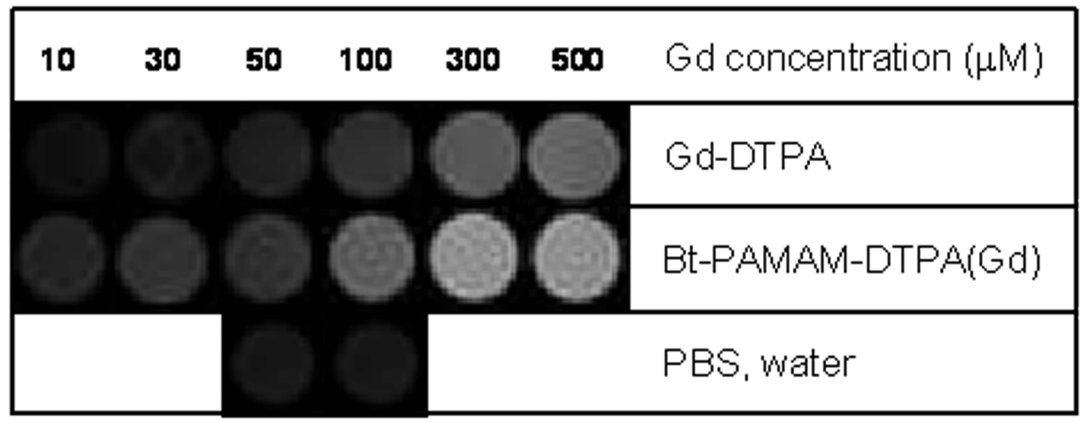

(b)

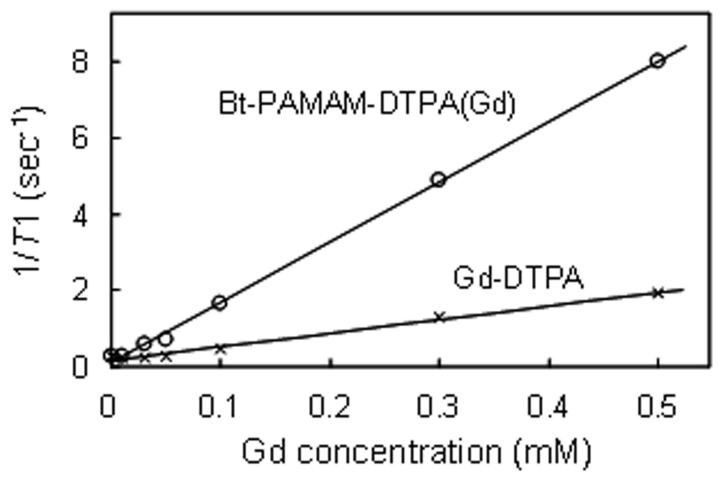




\section{\{s\} MTT-MMP}

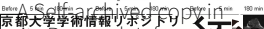

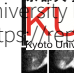

(b)

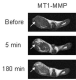

Negave contral

Salne

(c)
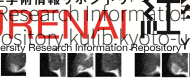

IC
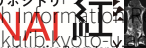

$4 \sqrt{2}$
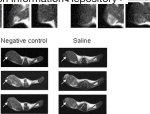

(d)
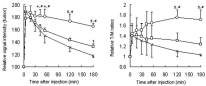

(e)

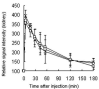

$$
\begin{aligned}
& \text {-0- MTs aes } \\
& \rightarrow 0-\text { Megave coetrol } \\
& \text { w- Sine }
\end{aligned}
$$

